\title{
Violencia obstétrica: conducta del personal médico y percepción de las usuarias en las salas de parto. Estudio multicéntrico, estado Anzoátegui *
}

\author{
Drs. (D) Santiago Rodríguez-Roque1, (DDinora Rodríguez-Rico¹, (D) Gloria Rodríguez-Rico ${ }^{1}$ \\ (iD) Rangel Jiménez-Malavé ${ }^{1}$.
}

\begin{abstract}
RESUMEN
Objetivo: Relacionar la percepción que tienen las usuarias embarazadas ante la violencia obstétrica en función a la conducta del personal médico en sala de partos, en tres centros de salud del estado Anzoátegui, durante octubre de 2018.

Métodos: Estudio de campo, no experimental, correlacional, prospectivo y transversal cuya muestra fue de 564 pacientes y 70 médicos provenientes de 3 centros de salud del estado Anzoátegui utilizando un cuestionario como instrumento de recolección de datos.

Resultados: Los médicos evaluados poseen un conocimiento regular y una conducta deficiente (60\% de los casos) sin relación entre sí $(p>0,05)$. Los tipos de trato deshumanizante principales fueron: estar en posición obligada y la realización de procedimientos sin autorización (99,29\% y 73,36\% de los casos). El tipo de violencia que predominó fue la fisica en el $100 \%$ de los casos, seguida del binomio fisico-psicológico, sin embargo la percepción de la paciente sobre la conducta del médico fue buena $(75,2 \%)$.

Conclusiones: Según el trato deshumanizante percibido por la usuaria en los tres centros de salud, se obtuvo que el índice de violencia obstétrica fue del $100 \%$, puesto que todas percibieron al menos un tipo de violencia.
\end{abstract}

Palabras clave: Obstetricia, Violencia, Derechos de la mujer, Abuso de los derechos humanos, Mujeres embarazadas.

\section{SUMMARY}

Objective: To relate the perception that pregnant users have about obstetric violence based on the behavior of medical staff in the delivery room at three health centers in Anzoátegui state during October 2018.

Methods: Non-experimental, correlational, prospective and cross-sectional field study with a sample of 564 patients and 70 physicians from 3 health centers in Anzoátegui state, using a questionnaire as a data collection instrument.

Results: The evaluated doctors have regular knowledge and poor behavior (60\% of cases) unrelated to each other ( $p$ : $>0.05)$. The main types of dehumanizing treatment were: being in a mandatory position and performing procedures without authorization (99.29\% and $73.36 \%$ of cases). The type of violence that prevailed was physical in $100 \%$ of cases, followed by physical-psychological binomial, however the patient's perception of the doctor's behavior was $\operatorname{good}(75.2 \%)$.

Conclusions: According to the dehumanizing treatment perceived by the user in the three health centers, it was obtained that the obstetric violence rate was $100 \%$ since all perceived at least one type of violence.

Keywords: Obstetrics, Violence, Women's Rights, Human Rights Abuses, Pregnant Women.

\begin{abstract}
${ }^{1}$ Universidad de Oriente, Núcleo Anzoátegui, Facultad de Medicina. Venezuela. *Trabajo de Grado presentado ante la Universidad de Oriente como requisito parcial para optar al título de Médico Cirujano obteniendo calificación de aprobado con mención honorifica y mención publicación. No se obtuvo financiamiento por parte de terceros para la realización de la investigación.
\end{abstract}

\section{INTRODUCCIÓN}

Los derechos humanos son facultades y atribuciones que emergen de la esencia de la persona, cuya condición se erige en el valor de la dignidad (1). Venezuela es uno de los países que contempla los derechos humanos como derecho fundamental (2). En la actualidad se han 
tipificado diferentes abusos a los derechos humanos, dentro de los cuales está la violencia contra la mujer, siendo considerado como todo ataque material y simbólico que discrimine, intimide, ignore, someta y subordine a las mujeres en los diferentes aspectos de su existencia $(3,4)$.

Dentro de los tipos de violencia contra la mujer está la violencia obstétrica (VO), que incluye cualquier acción que produce efectos negativos de carácter físico y psicológico, durante el proceso de parto (5). Su materialización se produce a través de un trato deshumanizado que surge principalmente de profesionales de la salud y pone de manifiesto una relación asimétrica entre la mujer y estos, revelando una desigualdad, tanto simbólica como real que dificulta el cumplimiento de los derechos básicos de la gestante (6).

En 2007, Venezuela fue pionera en publicar una herramienta legal para seguir trabajando en pro de la mujer y de su bienestar, convirtiéndose en el primer país en definir legalmente VO y en tipificarla como delito (7), cuando se promulga la Ley Orgánica Sobre el Derecho de las Mujeres a una Vida Libre de Violencia (LODMVLV), definiéndola como "la apropiación del cuerpo y procesos reproductivos de las mujeres por el personal de salud, que se expresa en un trato deshumanizador, en abuso de medicalización y patologización de los procesos naturales..." (8) estipulando los actos considerados como tal y su repercusión legal.

A pesar que la LODMVLV está vigente desde 2007, hoy día se puede confirmar que tanto el personal de salud que labora en los hospitales y maternidades del país, así como la población en general desconocen la mencionada ley, lo que trae como consecuencia que la violación de los derechos de las mujeres se siga perpetuando por desconocimiento, lo que hace importante establecer si la mencionada ley, realmente se aplica en el ejercicio de la medicina, después de doce años de haber sido promulgada.
Se debe resaltar, la poca difusión y enseñanza de la LODMVLV en el personal de salud y que el estudio de los posibles actos de $\mathrm{VO}$ en los centros hospitalarios venezolanos no ha recibido suficiente atención dentro del contexto social del país y, como consecuencia del manejo inadecuado del tema, pasa inadvertida la grave situación y repercusión moral, ética y legal que esto representa.

Es importante destacar que no se han publicado hasta la actualidad trabajos que evalúen el comportamiento de la VO en los hospitales públicos del estado Anzoátegui, lo que constituye un marco referencial para evaluar la atención hospitalaria en esta zona. La importancia de este estudio radica en poner en perspectiva la VO y abordarla como un problema de salud pública, por lo que se plantea relacionar la percepción que tienen las usuarias embarazadas ante la VO en función a la conducta del personal médico en sala de partos en tres centros de salud del estado Anzoátegui, durante octubre de 2018.

\section{MÉTODOS}

Se realizó un estudio multicéntrico en los siguientes centros de salud del estado Anzoátegui: Hospital Universitario "Dr. Luis Razetti” (HULR), Instituto Venezolano de los Seguros Sociales "Dr. Domingo Guzmán Lander" (IVSS HDGL) e Instituto Venezolano de los Seguros Sociales “Dr. César Rodríguez" (IVSS HCR).

La población la conformaron dos grupos, uno constituido por todas las usuarias que acudieron al Servicio de Sala de Partos y el otro por el personal médico que labora en los centros de salud mencionados, durante octubre de 2018. Esta información fue aportada por la oficina de epidemiología, registros médicos y dirección del departamento.

La población de mujeres se obtuvo estableciendo un 
promedio mensual del total de usuarias atendidas en el periodo de enero a septiembre de 2018, obteniéndose: a. HULR: 450. b. IVSS HDGL: 498. c. IVSS HCR: 213. La población de médicos se obtuvo por la totalidad de los trabajadores en el servicio de ginecología y obstetricia en los tres centros de salud, siendo: a. HULR: 9 residentes de primer año, 9 residentes de segundo año, 12 residentes de tercer año y 22 adjuntos. b. IVSS HDGL: 6 residentes asistenciales y 12 adjuntos. c. IVSS HCR: 4 residentes asistenciales y 12 adjuntos.

La muestra de las usuarias se obtuvo por muestreo probabilístico para cada centro de salud, utilizando la fórmula de muestreo probabilístico para población finita (9) Para la determinación de la muestra del personal médico se utilizó el método de muestreo no probabilístico, intencional partiendo de los siguientes criterios de inclusión: a. Trabajar como médico en sus diferentes categorías dentro del centro de salud en el Servicio de Sala de Parto. b. Encontrarse activo durante el periodo designado para la recolección de datos. c. Aceptar voluntariamente al estudio. d. Firmar el consentimiento informado.

Se utilizó como instrumento dos cuestionarios ad hoc validados a través del juicio de expertos, el primero se aplicó a las usuarias a través de cuatro secciones que establecen características sociodemográficas y ginecobstétricas. Asimismo, preguntas cerradas que evalúan la percepción del trato en el Servicio de Sala de Parto, por último, se realizaron preguntas para calificar en qué medida poseen conocimiento de la LODMVLV y sus derechos a ser atendidas sin VO.

El segundo cuestionario se aplicó al personal médico con la finalidad de evaluar los conocimientos sobre VO y la conducta de estos en la atención de la embarazada mediante dos secciones específicas y una sección que permite agruparlos según sus características sociodemográficas. Las preguntas contenidas en ambos instrumentos fueron basadas en la LODMVLV.
Antes de aplicarlo se obtuvo la autorización del director de cada centro de salud y se solicitó el consentimiento informado. Los datos obtenidos con referencia al conocimiento, percepción y conducta fueron agrupados en una escala de evaluación que va de cero (0) a diez (10) puntos con un mínimo aprobatorio de cinco (5) puntos, esta fue extrapolada a una escala cualitativa donde se utilizaron los siguientes criterios: insuficiente o deficiente (según pertinencia) de 0 a 4 puntos, regular de 5 a 6 puntos, bueno de 7 a 8 puntos y excelente de 9 a 10 puntos.

Los datos fueron procesados en el programa SPSS 24.0. Se calculó frecuencia y porcentaje de las variables categóricas; media, desviación estándar y mínimos y máximos de las variables continuas. Se utilizó la prueba de Chi-cuadrado de Pearson y Rho de Spearman para determinar $\mathrm{p}$ valor. La validez estadística se ubicó en $\mathrm{p}<0,05$.

\section{RESULTADOS}

Se encuestaron 70 médicos distribuidos de la siguiente forma: a. HULR: 11 médicos adjuntos (15,71\%), 12 médicos de tercer año $(17,14 \%), 9$ médicos de segundo año $(12,86 \%)$ y 9 médicos de primer año $(12,86 \%)$. b. IVSS HDGL: 5 médicos asistenciales $(7,14 \%)$ y 11 médicos adjuntos $(15,71 \%)$ y c. IVSS HCR: 4 médicos asistenciales $(5,72 \%)$ y 9 médicos adjuntos $(12,86 \%)$. La edad predominante se encuentra entre los 20 y 30 años $(51,42 \%)$ con una media de 33,64 \pm 7,305 años (mín. 25 y máx. 56), entre ellos, el sexo predominante fue el femenino $(\mathrm{p}>0,05)$ (Tabla 1$)$.

En cuanto a los conocimientos de los médicos evaluados con respecto al tema de la VO, se encontró que estos eran principalmente de regular a insuficiente en los tres establecimientos estudiados $(\mathrm{p}>0,05)$ (Tabla 2). Se observó que el conocimiento aumentaba conforme a los años de preparación, consiguiendo la excelencia en su etapa como adjunto $(\mathrm{p}>0,05)$ (Tabla $3)$. 
Tabla 1. Características de los médicos por edad y sexo

\begin{tabular}{lcccc}
\hline \multirow{2}{*}{ Edad } & \multicolumn{3}{c}{ Sexo } \\
& \multicolumn{2}{c}{ Masculino } & \multicolumn{2}{c}{ Femenino } \\
\cline { 2 - 5 } & $\mathrm{n}$ & $\%$ & $\mathrm{n}$ & $\%$ \\
\hline $20-30$ & 8 & 44,4 & 28 & 53,8 \\
$31-40$ & 4 & 22,2 & 16 & 30,8 \\
$41-50$ & 5 & 27,8 & 6 & 11,5 \\
$>50$ & 1 & 5,6 & 2 & 3,8 \\
\hline
\end{tabular}

Chi-cuadrado de Pearson: 22,245 p=0,386

Tabla 2. Conocimiento del médico sobre la violencia obstétrica

\begin{tabular}{lcccccc}
\hline & \multicolumn{6}{c}{ Centro } \\
\cline { 2 - 7 } Conocimiento & HULR & \multicolumn{3}{c}{ IVSS HDGL } & \multicolumn{1}{c}{ IVSS HCR } \\
\cline { 2 - 7 } & $\mathrm{n}$ & $\%$ & $\mathrm{n}$ & $\%$ & $\mathrm{n}$ & $\%$ \\
\hline $\begin{array}{l}\text { Insuficiente } \\
(0-4)\end{array}$ & 10 & 24,4 & 1 & 6,3 & 3 & 23,1 \\
$\begin{array}{l}\text { Regular } \\
(5-6)\end{array}$ & 24 & 58,5 & 11 & 68,8 & 7 & 53,8 \\
$\begin{array}{l}\text { Bueno } \\
(7-8)\end{array}$ & 7 & 17,1 & 1 & 6,25 & 1 & 7,7 \\
$\begin{array}{l}\text { Excelente } \\
(9-10)\end{array}$ & 0 & 0 & 3 & 18,8 & 2 & 15,4 \\
\hline
\end{tabular}

Chi-cuadrado de Pearson: $10,832 \mathrm{p}=0,094$

HULR: Hospital Universitario "Dr. Luis Razetti". IVSS HDGL: Instituto Venezolano de los Seguros Sociales "Dr. Domingo Guzmán Lander". IVSS HCR: Instituto Venezolano de los Seguros Sociales "Dr. César Rodríguez".
De los actos que son considerados como VO, resaltó el hecho que todos los médicos hacen uso de oxitocina durante la atención de la embarazada en trabajo de parto; asimismo, 68 (97,1\%) no cumplen con la denuncia obligatoria ante los entes pertinentes en caso de ser testigos de este delito, 63 (90\%) hacen uso de la maniobra de Kristeller y 41 (58,6\%) emplean la fuerza física hacia la paciente (Tabla 4). Sin embargo, estos en su mayoría reconocen poseer una conducta de insuficiente a regular en la atención de la embarazada $(\mathrm{p}<0,05)$ (Tabla 5).

Cuando se estudió la relación existente entre el conocimiento de los médicos sobre el tema de la VO y su conducta en la atención de la paciente embarazada que acude al servicio de sala de parto, con una probabilidad de error del 5,41\%, se puede afirmar que no estuvieron asociadas; ya que a pesar del conocimiento sobre el tema se cometió brusquedad en la acción; por el contrario, con poco conocimiento el trato fue adecuado (Rho de Spearman: 0,23120, $\mathrm{p}=0,05413$ ) (Figura 1).

En cuanto a las mujeres encuestadas, la edad predominante se encontró entre los 21 a 30 años con una media de 25,71 \pm 5,57 años (mínimo 18 y máximo 46). Asimismo, la mayoría tenía pobreza relativa (estrato IV según Graffar) y mayoritariamente poseían la secundaria aprobada (Tabla 6). De estas, todas

Tabla 3. Conocimiento del médico sobre la violencia obstétrica según cargo

\begin{tabular}{lcccccccccc}
\hline & \multicolumn{10}{c}{ Centro } \\
\cline { 2 - 11 } Conocimiento & \multicolumn{1}{c}{ RA } & \multicolumn{1}{c}{ R1 } & \multicolumn{2}{c}{ R2 } & \multicolumn{2}{c}{ R3 } & \multicolumn{2}{c}{ Adjunto } \\
\cline { 2 - 11 } & $\mathrm{n}$ & $\%$ & $\mathrm{n}$ & $\%$ & $\mathrm{n}$ & $\%$ & $\mathrm{n}$ & $\%$ & $\mathrm{n}$ & $\%$ \\
\hline Insuficiente & 3 & 33,3 & 5 & 55,6 & 1 & 11,1 & 2 & 16,7 & 3 & 9,7 \\
Regular & 6 & 66,6 & 4 & 44,4 & 5 & 55,6 & 8 & 66,7 & 19 & 61,3 \\
Bueno & 0 & 0 & 0 & 0 & 3 & 33,3 & 2 & 16,7 & 4 & 12,9 \\
Excelente & 0 & 0 & 0 & 0 & 0 & 0 & 0 & 0 & 5 & 16,1 \\
\hline
\end{tabular}

Chi-cuadrado de Pearson: 20,794 p=0,05347

RA: Residente Asistencial. R1: Residente de primer año. R2: Residente de segundo año. R3: Residente de tercer año. 
Tabla 4. Actos de violencia obstétrica de acuerdo a la conducta del médico

\begin{tabular}{lcc}
\hline Actos de violencia obstétrica & $\mathrm{n}$ & $\%$ \\
\hline Indicación de oxitocina & 70 & 100 \\
$\begin{array}{l}\text { No cumple con denunciar casos de } \\
\text { violencia obstétrica }\end{array}$ & 68 & 97,1 \\
Utiliza la maniobra de Kristeller & 63 & 90,0 \\
$\begin{array}{l}\text { No permite la libre deambulación o } \\
\text { sedestación }\end{array}$ & 50 & 71,4 \\
No facilita el apego precoz & 41 & 58,6 \\
Uso de fuerza física & 41 & 58,6 \\
No ofrece distintas posiciones para el parto & 41 & 58,6 \\
Omite el consentimiento informado & 34 & 48,6 \\
$\begin{array}{l}\text { Omite información a la paciente acerca del } \\
\text { caso }\end{array}$ & 18 & 25,7 \\
\hline
\end{tabular}

Tabla 5. Conducta percibida por el médico en la atención de la embarazada

\begin{tabular}{lcccccc}
\hline & \multicolumn{6}{c}{ Centro } \\
\cline { 2 - 7 } Conocimiento & \multicolumn{1}{c}{ HULR } & \multicolumn{3}{c}{ IVSS HDGL } & \multicolumn{1}{c}{ IVSS HCR } \\
\cline { 2 - 7 } & $\mathrm{n}$ & $\%$ & $\mathrm{n}$ & $\%$ & $\mathrm{n}$ & $\%$ \\
\hline $\begin{array}{l}\text { Insuficiente } \\
(0-4)\end{array}$ & 18 & 43,9 & 12 & 75,0 & 12 & 92,3 \\
$\begin{array}{l}\text { Regular } \\
(5-6)\end{array}$ & 12 & 29,3 & 4 & 25,0 & 0 & 0,0 \\
$\begin{array}{l}\text { Bueno } \\
(7-8)\end{array}$ & 10 & 24,4 & 0 & 0,00 & 1 & 7,7 \\
$\begin{array}{l}\text { Excelente } \\
(9-10)\end{array}$ & 1 & 2,4 & 0 & 0,0 & 0 & 0,0 \\
\hline \multicolumn{1}{c}{ Chi-cuadrado de Pearson: $14,091 \mathrm{p}=0,029$} \\
\end{tabular}

HULR: Hospital Universitario "Dr. Luis Razetti". IVSS HDGL: Instituto Venezolano de los Seguros Sociales "Dr. Domingo Guzmán Lander". IVSS HCR: Instituto Venezolano de los Seguros Sociales "Dr. César Rodríguez".

Figura 1. Influencia del conocimiento sobre la violencia obstétrica en la conducta del médico ante la atención de la embarazada

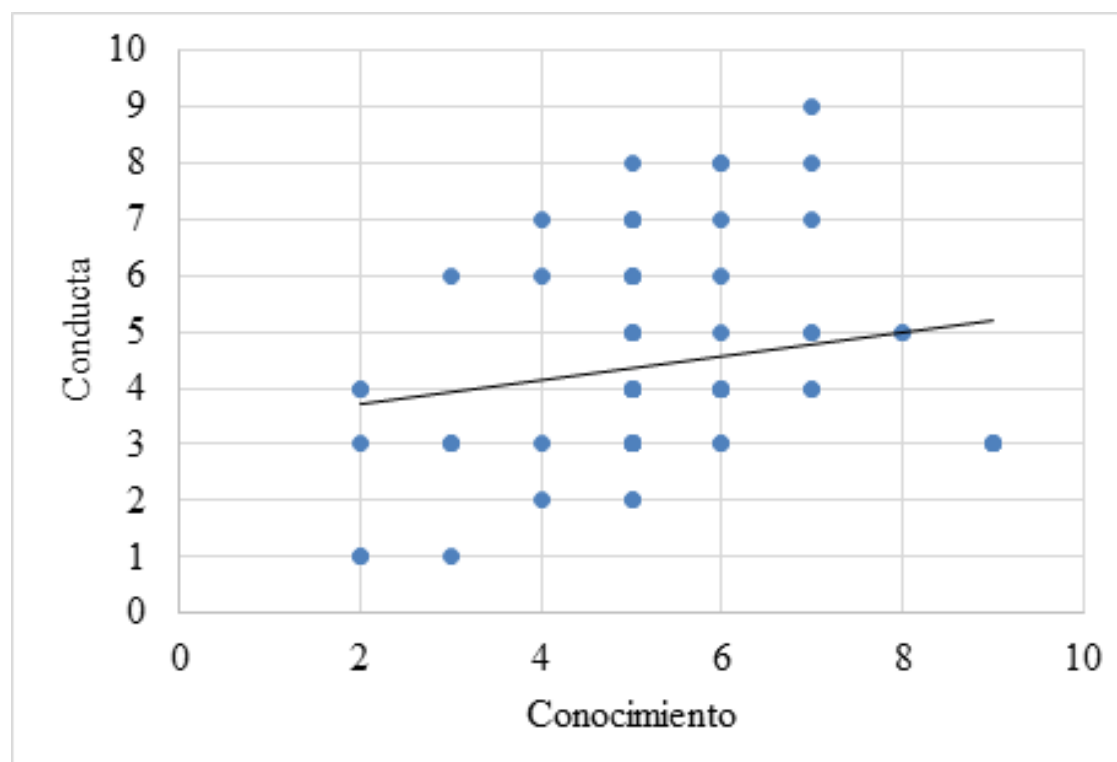

$\mathrm{Y}=0,2163 \mathrm{x}+3,2759 \mathrm{R} 2=0,0397$ 
VIOLENCIA OBSTÉTRICA: CONDUCTA DEL PERSONAL MÉDICO Y PERCEPCIÓN DE LAS

USUARIAS EN LAS SALAS DE PARTO. ESTUDIO MULTICÉNTRICO, ESTADO ANZOÁTEGUI

Tabla 6. Características generales de las usuarias en estudio

\begin{tabular}{lcccccc}
\hline Característica & \multicolumn{5}{c}{ Centro de salud } \\
\cline { 2 - 7 } & \multicolumn{2}{c}{ HULR } & \multicolumn{2}{c}{ IVSS HDGL } & \multicolumn{2}{c}{ IVSS CR } \\
\cline { 2 - 7 } & $\mathrm{n}$ & $\%$ & $\mathrm{n}$ & $\%$ & $\mathrm{n}$ & $\%$ \\
\hline Edad & & & & & & \\
$11-20$ & 50 & 8,87 & 47 & 8,33 & 42 & 7,45 \\
$21-30$ & 111 & 19,68 & 109 & 19,33 & 70 & 12,41 \\
$31-40$ & 45 & 7,98 & 61 & 10,82 & 23 & 4,08 \\
$>40$ & 2 & 0,35 & 1 & 0,18 & 3 & 0,53 \\
Graffar & & & & & & \\
II & 0 & 0,00 & 3 & 0,53 & 0 & 0,00 \\
III & 62 & 10,99 & 57 & 10,11 & 45 & 7,98 \\
IV & 123 & 21,81 & 135 & 23,94 & 76 & 13,48 \\
V & 23 & 4,08 & 23 & 4,08 & 17 & 3,01 \\
Instrucción & & & & & & \\
Ninguno & 2 & 0,35 & 0 & 0,00 & 0 & 0,00 \\
Primaria & 62 & 10,99 & 64 & 11,35 & 31 & 5,50 \\
Secundaria & 111 & 19,68 & 109 & 19,33 & 75 & 13,30 \\
Superior & 33 & 5,85 & 45 & 7,98 & 32 & 5,67 \\
\hline
\end{tabular}

Edad: Chi-cuadrado de Pearson 40,395 p=0,832. Graffar: Chi-cuadrado de Pearson 6,946 p=0,325. Instrucción: Chi-cuadrado de Pearson: 8,219 $\mathrm{p}=0,2224$

Tabla 7. Tipo de violencia obstétrica generada a la embarazada

\begin{tabular}{lcccccc}
\hline \multirow{2}{*}{$\begin{array}{l}\text { Tipo de } \\
\text { maltrato }\end{array}$} & \multicolumn{6}{c}{ Centro } \\
\cline { 2 - 7 } & HULR & \multicolumn{2}{c}{ IVSS HDGL } & \multicolumn{2}{c}{ IVSS HCR } \\
\cline { 2 - 7 } & $\mathrm{n}$ & $\%$ & $\mathrm{n}$ & $\%$ & $\mathrm{n}$ & $\%$ \\
\hline $\begin{array}{l}\text { Psicológico } \\
\text { (F) }\end{array}$ & 140 & 24,8 & 125 & 22,2 & 78 & 13,8 \\
Mixto* & 50 & 8,9 & 76 & 13,5 & 46 & 8,2 \\
Físico & 12 & 2,1 & 9 & 1,6 & 7 & 1,2 \\
Verbal (F) & 6 & 1,1 & 8 & 1,4 & 7 & 1,2 \\
\hline
\end{tabular}

* Presencia de los tres tipos en la misma embarazada

HULR: Hospital Universitario "Dr. Luis Razetti”. IVSS HDGL: Instituto Venezolano de los Seguros Sociales "Dr. Domingo Guzmán Lander". IVSS HCR: Instituto Venezolano de los Seguros Sociales "Dr. César Rodríguez". manifestaron $\mathrm{VO}$, maltrato físico en combinación con el de tipo psicológico, en su mayoría (Tabla 7); las principales causas fueron la obligada posición para parir (560 pacientes/99,3\%), la realización de procedimientos sin autorización expresa de la paciente (416 pacientes/73,8 \%) y la demora en la atención (377 pacientes/66,8 \%) (Tabla 8 ).

A pesar de estos resultados, la percepción de las pacientes con respecto a la conducta del personal médico en la sala de partos fue considerada principalmente buena en los tres centros de salud del estado, con valores atípicos en los IVSS (18\%) y en el HULR $(8,7 \%)(p<0,05)$ (Tabla 9), siendo esta percepción mayor positivamente con respecto a la conducta reconocida por el médico tratante (diferencia estimada de media entre 1,86 y 2,78 ) (Figura 2). 
Tabla 8. Trato deshumanizante percibida por la usuaria

\begin{tabular}{lcc}
\hline Tipo de violencia obstétrica & $\mathrm{n}$ & $\%$ \\
\hline $\begin{array}{l}\text { Posición obligada durante el trabajo de } \\
\text { parto }\end{array}$ & 560 & 99,29 \\
Procedimiento sin autorización & 416 & 73,76 \\
Demora en la atención & 377 & 66,84 \\
Información insuficiente & 270 & 47,87 \\
Falta de apego precoz* $(\mathrm{n}=524)$ & 193 & 36,83 \\
Malas respuestas & 141 & 25,00 \\
Falta de respeto & 115 & 20,39 \\
Actitud de desprecio & 111 & 19,68 \\
Regaños y amenazas & 69 & 12,23 \\
Críticas por su comportamiento & 58 & 10,28 \\
Sufrió agresión física directa & 19 & 3,37 \\
\hline
\end{tabular}

Con referencia al conocimiento sobre el derecho a ser atendidas sin sufrir VO se encontró que casi la totalidad del conocimiento se ubicó dentro de los niveles de nada $(35,46 \%)$ y muy poco $(51,60 \%)$, las
Tabla 9. Percepción de las embarazadas sobre la conducta del personal médico

\begin{tabular}{lcccccc}
\hline & \multicolumn{6}{c}{ Centro } \\
\cline { 2 - 7 } Conocimiento & \multicolumn{1}{c}{ HULR } & \multicolumn{3}{c}{ IVSS HDGL } & \multicolumn{2}{c}{ IVSS HCR } \\
\cline { 2 - 7 } & $\mathrm{n}$ & $\%$ & $\mathrm{n}$ & $\%$ & $\mathrm{n}$ & $\%$ \\
\hline $\begin{array}{l}\text { Deficiente } \\
(0-4)\end{array}$ & 18 & 8,7 & 40 & 18,3 & 26 & 18,8 \\
$\begin{array}{l}\text { Regular } \\
(5-6)\end{array}$ & 12 & 5,8 & 22 & 10,1 & 12 & 8,7 \\
$\begin{array}{l}\text { Bueno } \\
(7-8)\end{array}$ & 174 & 83,7 & 152 & 69,7 & 98 & 71,0 \\
$\begin{array}{l}\text { Excelente } \\
(9-10)\end{array}$ & 4 & 1,9 & 4 & 1,8 & 2 & 1,4 \\
\hline
\end{tabular}

Chi-cuadrado de Pearson: 14,41274 p=0,0253

HULR: Hospital Universitario "Dr. Luis Razetti". IVSS HDGL: Instituto Venezolano de los Seguros Sociales "Dr. Domingo Guzmán Lander". IVSS HCR: Instituto Venezolano de los Seguros Sociales "Dr. César Rodríguez".

restantes manifestaron poseer mucho conocimiento al respecto (n:564). Por su parte, solo el $33 \%$ (186) de las encuestadas conocía la LODMVLV y el 13,29\% (75) conocía los mecanismos de denuncia.

Figura 2. Trato percibido por las usuarias sobre la conducta del personal médico

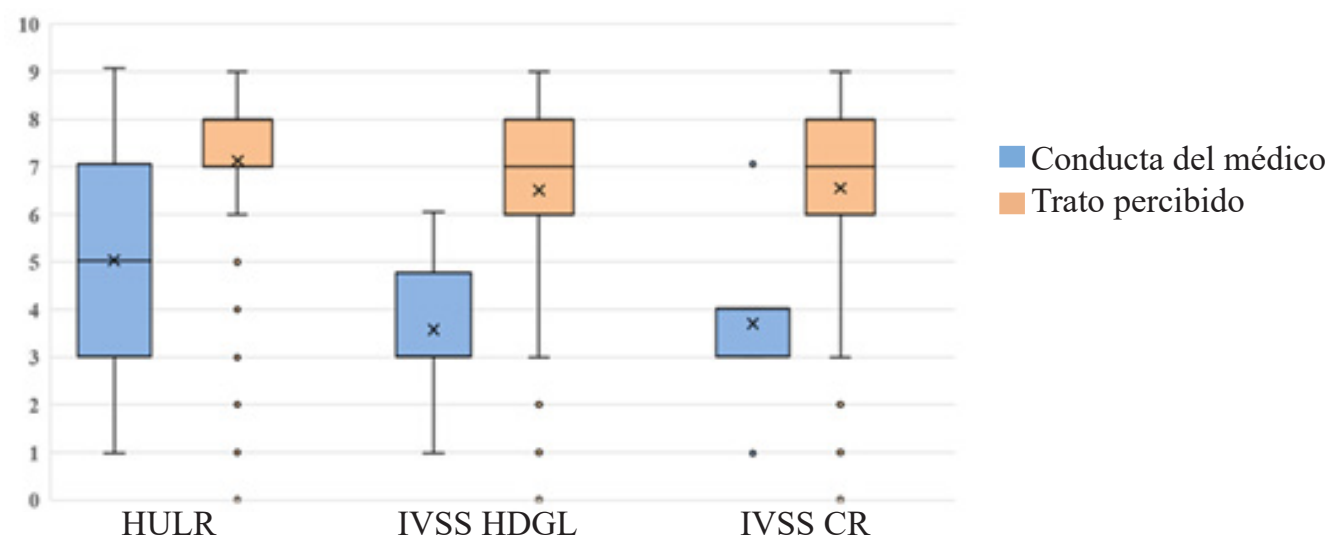

HULR: Hospital Universitario "Dr. Luis Razetti”. IVSS HDGL: Instituto Venezolano de los Seguros Sociales "Dr. Domingo Guzmán Lander". IVSS HCR: Instituto Venezolano de los Seguros Sociales "Dr. César Rodríguez”. 


\section{DISCUSIÓN}

La violencia obstétrica constituye un importante problema en el marco de los derechos de las mujeres. De acuerdo a los resultados presentados se puede afirmar que se trata de un problema que aún no está controlado y que puede generar muchos matices de opinión. Según los resultados mostrados, el nivel de conocimiento del médico sobre VO aumentó a medida que adquieren más experiencia, pudiendo ser explicado por la sobrecarga de trabajo, la cual va disminuyendo con la trayectoria profesional (10). A pesar de esto, el conocimiento fue inferior a lo ya reportado (11). Cabe resaltar que no existe, hasta la fecha, más investigaciones con las que comparar el conocimiento del médico sobre el tema en el estado Anzoátegui.

Según la conducta, todos los médicos incurrieron en $\mathrm{VO}$, principalmente por usar oxitócicos durante su atención $(100 \%)$ sin solicitar el consentimiento informado, hecho que se asemeja a la conducta del $80 \%$ y $85,71 \%$ de los médicos, expuesta en otros estudios $(4,12)$. Es frecuente que los médicos alteren el proceso natural del parto mediante el uso de técnicas de aceleración, lo que pone a dichas mujeres en una situación de riesgo a desarrollar complicaciones por el uso del mismo $(11,13)$. Este aspecto ha generado incomodidad entre los médicos por cuanto los oxitócicos pueden ser de gran ayuda en la atención del trabajo de parto (14). La Organización Mundial de la Salud (OMS) expresa que no está claro que el uso de la oxitocina ofrezca algún beneficio a las mujeres o a los fetos y recomienda que se debe suministrar con una indicación médica precisa $(15,16)$.

A pesar que la LODMVLV está vigente desde hace 13 años; la mayoría de los médicos evaluados la desconocen, así como tampoco denuncian actos de VO. Este hecho se ha perpetuado en el tiempo, según lo reportado por Faneite y col. (11) quien refiere que el $72,6 \%$ de los médicos desconocen los mecanismos de denuncia y el 71,8 \% desconoce los organismos encargados de prestar ayuda.

Con relación a las conductas de VO, el uso de fuerza física directa contra la parturienta refleja que el valor está por encima de lo esperado si se considera que este debería ser de cero. En la mayoría de los casos se realiza la maniobra de Kristeller, en alrededor del $60,71 \%$ de los casos (14). La OMS y la Declaración Universal de los Derechos Humanos consideran esta maniobra como una conducta contraria al parto, que vulnera el respeto a la dignidad e integridad de la mujer sobre su cuerpo (12). A nivel nacional e internacional, no existe una estadística de frecuencia con la cual comparar estas cifras. Se presume que pudiera ser por las implicaciones legales que pudiera conllevar, ya que es una violación grave a la integridad física de la mujer contemplada en la LODMVLV (12).

Con relación a otras formas de $\mathrm{VO}$, debe hacerse la salvedad que no ofrecer distintas posiciones para el parto se debe a que en los tres centros de salud evaluados no existen salas de parto acondicionadas que permitan otras modalidades de atención a la conocida posición supina. Además de esto, la enseñanza de la obstetricia y ginecología en Venezuela no contempla, el parto vertical por lo que hay experiencia limitada acerca de su uso $(14,17,18)$. El apego precoz no fue promovido siendo que el primer contacto madre-hijo debe hacerse de inmediato, la liberación de hormonas produce el apego y resulta eficiente y exitoso el proceso de lactancia materna, además el contacto visual y táctil en los primeros minutos de vida marcan el vínculo afectivo entre ambos para toda la vida (19).

Todas las usuarias estudiadas tuvieron algún tipo de maltrato físico directo o combinado, dejando en evidencia el alto índice de violencia, esto se contrapone a lo reportado en otros estudios donde el $25 \%$ (20) y el $29 \%$ (21) de las entrevistadas refieren haber sido violentadas, desde violencia verbal (palabras ofensivas o despectivas hasta frases como 
"no grite", "no llore", "no se queje"), a física (sutura sin anestesia, golpes en las piernas o pellizcos). Las mujeres salen con frecuencia traumatizadas de sus partos como consecuencia de estas acciones, incluso tienen recuerdos muy nítidos por años (22).

En el periodo prenatal, durante el parto y en el posparto la mujer necesita recibir apoyo de profesionales de salud capacitados y comprometidos con la fisiología del nacimiento, que respeten la gestación, el parto y la lactancia como procesos fisiológicos. Se ha demostrado que estas experiencias serían recordadas no solo por el momento singular en la vida de la madre y del hijo, sino también por los actos violentos y hechos traumáticos en los que la mujer se sintió agredida, irrespetada y violentada, pudiendo impedir incluso la posibilidad de relaciones sexuales y perjudicar la relación conyugal, además, este tipo de violencia tiene implicaciones sobre la morbimortalidad materna durante el parto y el posparto (20).

Todas las usuarias percibieron algún tipo de trato deshumanizante, estando muy por encima de lo ya reportado $(14,21)$. A casi la totalidad de las usuarias les realizaron algún tipo de procedimiento sin autorización expresa ni recibieron información suficiente y adecuada sobre estos. Terán y col. (14) publicaron que en el $66,8 \%$ manifestaron actos médicos sin consentimiento, mientras que Pereira y col. (4) reportaron que 34,4 \% no recibía información suficiente al respecto. Dichos datos son considerados alarmantes, sin embargo, resultan ser menores a los de este estudio.

La actitud del médico relacionada con la falta de respeto, desprecio, regaño, amenazas y críticas a las usuarias reflejan la baja sensibilidad sobre VO (14). A pesar que solo 19 usuarias $(3,37 \%)$ expresaron haber percibido agresión física directa por parte de médicos, sigue siendo un número considerable de casos a pesar que lo que se ha reportado hasta ahora ha sido solo del $0,9 \%$ al $8 \%$ a nivel nacional $(4,20)$.
Es importante destacar que la mayoría categorizó la atención medica como buena en los tres centros de salud estudiados (75,2 \%), hecho que se asemeja a lo reportado en otros estudios con un $89 \%$ de aceptación (21). Es preocupante evidenciar una contradicción cuantitativa entre lo que las usuarias consideraron como un trato eficiente frente a la percepción deficiente del médico en su conducta y el conocimiento en VO; esto pudiera denotar que las usuarias de la sala de parto están acostumbradas a recibir un trato inadecuado y no considerarlo de ese modo. Esta percepción pudiera ser explicada porque las mujeres actuales están más permisibles a conductas médicas innecesarias y que algunas incluso las desean por haber introducido culturalmente un modelo de asistencia que se ofrece de forma hegemónica (20). También se puede inferir que esta percepción se deba al hecho que casi la totalidad de las usuarias estudiadas poseen un escaso conocimiento sobre violencia obstétrica pudiendo no reconocerla. Este hecho se observó también en lo publicado por Pereira y col. (4). La violencia consensual se ha propuesto que nace en el consentimiento de las mujeres, que buscan justificar la agresividad y las dificultades experimentadas como parte inherente del proceso de nacimiento y parto, y en la invisibilidad a los ojos del profesional de la salud, que cree que la violencia es solo un caso aislado y que no sucede en grandes proporciones (23).

Los factores relacionados con la ocurrencia de la VO fueron como causa predominante, la conducta tomada por el personal médico y su conocimiento ya que tratan con brusquedad a las usuarias en las salas de parto. Además, la práctica sin recomendación de la medicalización excesiva, negligencia, privación de asistencia y maltrato, la limitación institucional para recibir a las gestantes con ambientes incómodos y desestructurados y un ritmo de trabajo alienante asociado a la precariedad de los recursos (20), influyen en la concepción de VO. Sin embargo, las condiciones de trabajo difíciles no se pueden tener como justificación para la práctica de VO (22). 
Es importante resaltar que a pesar de contar con la normativa legal que penaliza las prácticas estipuladas como VO y que permite a las mujeres empoderarse de su salud sexual y reproductiva, la mayoría de las usuarias desconoce sus derechos y cómo ejercerlos; a pesar que han transcurrido 13 años desde la promulgación de la ley, solo un pequeño grupo de mujeres posee información de esta, dejando al grupo que no tiene conocimiento sobre la misma vulnerables a ser violentadas (14).

La difusión a toda la población de la mencionada ley, así como los planes de capacitación e información, es responsabilidad del Ministerio del Poder Popular para la Salud, conjuntamente con el Instituto Nacional de la Mujer; a pesar de que sea algo obligatorio y necesario, las cifras demostradas en este y otros estudios citados reflejan que no se hace adecuadamente. Ha sido la Sociedad de Obstetricia y Ginecología de Venezuela, la que, en sus actividades científicas, ha dado a conocer lo referente al tema y a pesar de esto no ha sido suficiente (11).

Es evidente la urgente necesidad de difundir y hacer cumplir los derechos sexuales y reproductivos de las mujeres. En tanto la institución y las autoridades de salud no regulen la realización de estas prácticas y maniobras, el atropellamiento de los derechos humanos de las mujeres seguirá siendo una práctica naturalizada en la atención obstétrica (21). Es imperativo que el personal de salud que labora en estas áreas comprenda los alcances de la LODMVLV, las obligaciones que debe cumplir y las posibles penalidades en caso de infringirla, de igual forma las usuarias deben tener el conocimiento para hacer valer los derechos consagrados en ella (14).

A pesar de que la ley ha causado grandes expectativas y también ha generado dudas entre los médicos (11), aún persiste el incumplimiento en el personal de salud y desinformación en las usuarias. Muchos médicos argumentan que esta ley permite a las usuarias hacer peticiones sin fundamento, relacionadas con la vía del parto o negarse a determinados procedimientos, interfiriendo de esta forma en la adecuada ejecución de su trabajo, y piden al Estado realizar mejoras sustanciales en los hospitales que garanticen una óptima atención a las embarazadas (14). La OMS manifiesta que la falta de insumos e instalaciones inadecuadas es constitutivo de VO (24), lo que es importante y a la vez preocupante, dadas las condiciones recientes de la salud en Venezuela, con escasa dotación de insumos, deficiencia de personal y masiva asistencia de usuarias a los hospitales públicos (18) por lo que garantizar una atención libre de VO se hace una utopía.

Es necesario sensibilizar al personal de salud y a la comunidad en torno a la $\mathrm{VO}$ promoviendo una formación que aborde tanto el enfoque fisiológico del nacimiento como lo contextos culturales, sociales y psicológicos en los que está inmersa la mujer. Se insta a las autoridades competentes en materia de salud a equipar los centros de atención que permitan la atención de un parto humanizado, pues para la fecha de realización de la presente investigación, ninguno de los hospitales incluidos en este estudio contaba con áreas equipadas, ni habilitadas para la atención de esta modalidad de parto.

\section{REFERENCIAS}

1. Morales H. Introducción: notas sobre la transición en México y los derechos humanos. En: Derechos humanos: dignidad y conflicto. México: Editorial Universidad Interamericana; 1996. p. 19.

2. Constitución de la República Bolivariana de Venezuela. Gaceta Oficial de la República Bolivariana de Venezuela $\mathrm{N}^{\mathrm{o}} 5453$ (extraordinario) (24 de marzo de 2000).

3. Albarrán-C Y. Normativa jurídica en violencia contra las mujeres en Venezuela. Comunidad y Salud Maracay [Internet]. 2010 [consultado 10 de diciembre de 2019]; 8(2):79-82. Disponible en $\quad$ http://ve.scielo.org/scielo.php?script=sci arttext\&pid=S1690-32932010000200010

4. Pereira C, Domínguez A, Toro J. Violencia obstétrica 
desde la perspectiva de la paciente. Rev Obstet Ginecol Venez [Internet]. 2015 [consultado 10 de diciembre de 2019]; 75(2):81-90. Disponible en: $\quad$ http://ve.scielo.org/scielo.php?script=sci_ arttext\&pid=S0048-77322015000200002

5. Da-Silva, I. Formas de violencia obstétrica experimentada por madres que tuvieron un parto normal. Enferm glob [Internet] 2017 [consultado 10 de diciembre de 2019]; 16(47): 71-97. Disponible en: $\quad$ http://scielo.isciii.es/scielo.php?script $=$ sci arttext\&pid=S1695-61412017000300071

6. Posición de la Academia Nacional de Medicina sobre el tema de violencia obstétrica. Acta Méd Costarric [Internet] 2015 [consultado 10 de diciembre de 2019]; 57(3):143-144. Disponible en: https://www.scielo.sa.cr/scielo.php?script=sci arttext\&pid=S0001-60022015000300008

7. Belli L. La violencia obstétrica: otra forma de violación a los derechos humanos. Revista Redbioética [Internet]. 2013 [consultado 10 de diciembre de 2019]; 1(7):25-34. Disponible en: https://ri.conicet. gov.ar/bitstream/handle/11336/12868/Art2-BelliR7. pdf? sequence $=2$ \&isAllowed $=\mathrm{y}$.

8. Ley Orgánica Sobre el Derecho de las Mujeres A Una Vida Libre de Violencia. Gaceta Oficial de la República Bolivariana de Venezuela $N^{\circ} 38668$ (23 de marzo de 2015).

9. Castañeda J, De la Torre M, Moran J, Lara L. Metodología de la Investigación. México: Editorial Interamericana Mc Graw-Hill; 2002.

10. Pintado S, Penagos J. Casas M. Síndrome de desgaste profesional en médicos y percepción de la violencia obstétrica. Ginecol Obstet Mex [Internet] 2015 [consultado el 10 de diciembre de 2019]; 83(3):173178. Disponible en: https://www.medigraphic.com/ pdfs/ginobsmex/gom-2015/gom153f.pdf

11. Faneite J, Feo A, Toro J. Grado de conocimiento de violencia obstétrica por el personal de salud. Rev Obstet Ginecol Venez [Internet] 2012 [consultado 11 de diciembre de 2019]; 72(1):4-12. Disponible en: $\quad$ http://ve.scielo.org/scielo.php?script=sci arttext\&pid=S0048-77322012000100002

12. Farfán B. Parto humanizado: barreras y facilitadores según la percepción del personal médico [Trabajo Especial de Grado]. Caracas (DC): Universidad Central de Venezuela; 2017 [consultado 11 de diciembre de 2019]. Disponible en: http://saber.ucv.ve/ handle/123456789/16671.

13. Camacaro M, Ramírez M, Lanza L, Herrera M.
Conductas de rutina en la atención al parto constitutivas de violencia obstétrica. Utopía y Praxis Latinoam [Internet]. 2015 [consultado 11 de diciembre de 2019]; 20(68):113-120. Disponible en: https://www.redalyc. org/pdf/279/27937090009.pdf

14. Terán P, Castellanos C, González M, Ramos D. Violencia obstétrica: percepción de las usuarias. Rev Obstet Ginecol Venez [Internet]. 2013 [consultado 11 de diciembre de 2019]; 73(3):171-180. Disponible en: $\quad$ http://ve.scielo.org/scielo.php?script=sci arttext\&pid=S0048-77322013000300004

15. Lokugamage A, Pathberiya S. Human rights in childbirth, narratives and restorative justice: a review. Reprod Health [Internet]. 2017 [consultado 11 de diciembre de 2019]; 14(1):17. Disponible en: https:// www.ncbi.nlm.nih.gov/pmc/articles/PMC5289003/ pdf/12978 2016_Article 264.pdf

16. Organización Mundial de la Salud [Internet]. Ginebra: Cuidados en el parto normal: Una guía práctica; 1996 [consultado 11 de diciembre de 2019]. Disponible en: http://comatronas.es/contenidos/2012/03/textos/Guia practica_de_cuidados_en_el_parto_normal_OMS.pdf

17. Pérez R. Obstetric violence: A new legal term introduced in Venezuela, special editorial Int J Gynaecol Obstet [Internet]. 2010 [consultado 11 de diciembre de 2019]; 111(3):201-202. Disponible en: https:// obgyn.onlinelibrary.wiley.com/doi/epdf/10.1016/j. ijgo.2010.09.002

18. Pérez R. Cuatro años después de la promulgación de la Ley sobre el derecho de las mujeres a una vida libre de violencia. Rev Obstet Ginecol Venez [Internet]. 2011 [consultado 11 de diciembre de 2019]; 71(2):73-76. Disponible en: $\quad$ http://ve.scielo.org/scielo.php?script=sci arttext\&pid $=$ S0048-77322011000200001

19. Canelón M. Significado del parto vertical para los/ las obstetras. Una propuesta educativa [Trabajo Especial de Grado]. Valencia: Universidad de Carabobo; 2015 [consultado 11 de diciembre de 2019]. Disponible en: http://mriuc.bc.uc.edu.ve/bitstream/ handle/123456789/1292/malejandra.pdf?sequence=1.

20. Barros de Souza A, da Silva LC, das Neves Alves R, Jacinto Alarcão AC. Fatores associados à ocorrência de violência obstétrica institucional: uma revisão integrativa da literatura, Rev Ciênc Méd Campinas [Internet] 2016 [consultado 11 de diciembre de 2019]; 25(3):115-128. Disponible en: https://seer.sis.puccampinas.edu.br/seer/index.php/cienciasmedicas/ article/view/3641. 
21. Valdez-Santiago R, Hidalgo-Solórzano E, MojarroIñiguez M, Arenas-Monreal LM. Nueva evidencia a un viejo problema: el abuso de las mujeres en las salas de parto. Rev CONAMED [Internet] 2013 [Consultado 11 de diciembre de 2019]; 18(1):14 - 20. Disponible en: https://www.medigraphic.com/pdfs/conamed/con2013/con131c.pdf

22. Olza I. Estrés postraumático secundario en profesionales de la atención al parto, Aproximación al concepto de violencia obstétrica. C Med Psicosom [Internet]. 2014 [consultado 11 de diciembre de 2019]; 111:7983. Disponible en: https://dialnet.unirioja.es/servlet/ articulo? codigo $=4906961$. .

23. Oliveira VJ, Penna CMM. Discussing obstetric violence through the voices of women and health professionals.
Texto Context-enferm [Internet]. 2017 [consultado 11 de diciembre de 2019]; 26(2):1-10. Disponible en: http://dx.doi.org/10.1590/0104-07072017006500015

24. Castrillo B. Dime quién lo define y te diré si es violento, reflexiones sobre la violencia obstétrica. Sex Salud Soc [Internet]. 2016 [consultado el 11 de diciembre de 2019]; 24:43-68. Disponible en: https://www.scielo.br/ pdf/sess/n24/1984-6487-sess-24-00043.pdf

Recibido 29 de marzo de 2020 Aprobado 29 de abril de 2020 